Psychotherapeut 2022 $67: 135-142$

Angenommen: 10. Januar 2022

Online publiziert: 14. Februar 2022

(c) Der/die Autor(en) 2022

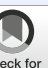

https://doi.org/10.1007/s00278-022-00574-1

\section{Wie wirkt das Miteinanderreden in Psychotherapien aus Sicht von Personen ohne Therapieerfahrung?}

\author{
Eine „Mixed-methods"-Studie
}

\author{
S. Wulf · F. Klapprott · B. Janning · A. Gumz \\ Fachbereich Psychosomatik und Psychotherapie, Psychologische Hochschule Berlin (PHB), Berlin, \\ Deutschland
}

\section{Zusammenfassung}

\section{In diesem Beitrag}
- Ziel der vorliegenden Studie
- Methoden
Studiendesign und Datenerhebung • Stichprobe - Instrumente und offene Fragestellung - Datenanalyse
- Ergebnisse
- Diskussion Interpretation der Ergebnisse - Me- thodische Stärken und Schwächen • Ausblick

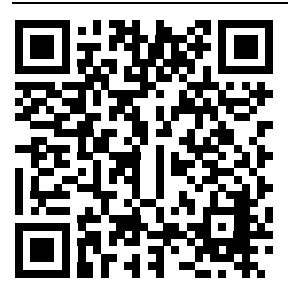

QR-Code scannen \& Beitrag online lesen
Hintergrund und Ziel: Das Miteinanderreden nimmt in Psychotherapien einen zentralen Raum ein - auch aus Sicht von Personen ohne Therapieerfahrungen. Es ist davon auszugehen, dass Personen ohne Therapieerfahrungen Ideen darüber entwickeln, auf welche Weise das therapeutische Miteinanderreden hilft. Diese Vorstellungen und Vorerwartungen können die therapeutische Interaktion in Psychotherapien beeinflussen. Um ein besseres Verständnis der Erwartungen an Prozesse in der Psychotherapie zu erhalten, soll untersucht werden, welches Bild sich Personen ohne Therapieerfahrungen vom Miteinanderreden in der Psychotherapie machen.

Material und Methode: Es handelt sich um eine "Mixed-methods"-Studie (qualitative Kategorienbildung mit anschließender quantitativer Häufigkeitsanalyse). An der querschnittlichen Onlineerhebung nahmen 225 Erwachsene ohne Therapie- oder Beratungserfahrung (Alter: Mittelwert $[M]=27,53$ Jahre, Standardabweichung [SD] \pm 9,93 Jahre, Range 19 bis 91 Jahre, Geschlecht: 207 weiblich, 18 männlich) teil. Neben soziodemografischen Daten wurden Antworten auf die offene Frage: „Wie und auf welche Art und Weise hilft das Miteinanderreden in der Psychotherapie?" erhoben und inhaltsanalytisch ausgewertet.

Ergebnisse: Die Teilnehmer:innen erwarteten Wirksamkeit des Miteinanderredens in der Psychotherapie in Bezug auf die 4 Hauptkategorien 1) Therapeutische Beziehung erleben, 2) Erleichterung verschaffen, 3) Erkenntnis gewinnen sowie 4) Veränderungen ermöglichen. Jeder dieser Oberkategorien wurden eine bis 8 Subkategorien zugeordnet. Besonders häufig wurden die Subkategorien „von der Seele reden/Ballast loswerden" und "Perspektivwechsel" genannt.

Diskussion: Die vorliegenden Ergebnisse deuten auf hin, dass Personen ohne Psychotherapieerfahrung differenzierte und jeweils individuell unterschiedliche Annahmen darüber haben, wie das Miteinanderreden in der Psychotherapie heilen kann. Ein Austausch darüber, mit welchen diesbezüglichen Erwartungen Patient:innen in eine Psychotherapie kommen, kann hilfreich sein.

\section{Schlüsselwörter}

Erwartungen · Redekur · Therapeutische Beziehung · Wirkfaktoren · Wirkmechanismen 
Traditionell gilt Psychotherapie als „talking cure", also als Behandlungsverfahren, das durch das Miteinanderreden Heilung ermöglicht. Mit welchen Erwartungen Personen der Psychotherapie begegnen, ist wichtig für die Auswahl von Behandlungsoptionen und den Verlauf von Therapien. Annahmen darüber, was Personen ohne Therapieerfahrung vom Miteinanderreden in der Psychotherapie erwarten, sind jedoch kaum erforscht. Ein besseres Verständnis von derartigen - gesellschaftlich vermittelten Annahmen über Psychotherapie kann hilfreich sein, um mit Erwartungen von Patient:innen und deren Angehörigen zielgerichtet zu arbeiten und potenziellen Missverständnissen vorzubeugen.

\section{Einleitung}

Psychotherapie kann nicht als losgelöst vom gesellschaftlichen Kontext gesehen werden, in dem die Therapie stattfindet (Avissar 2016). Soziale und mediale Repräsentationen beeinflussen bereits, ob Psychotherapie überhaupt in Erwägung gezogen wird, und welche Hilfsangebote als effektiv oder stigmatisiert wahrgenommen werden (Kushner und Sher 1991; Michalopoulou et al. 2017; Corrigan 2004; Tzur Bitan und Abayed 2020). Eine fehlende Passung von Erwartung und Therapiegeschehen kann sich zudem negativ auf die Zufriedenheit mit der Therapie auswirken (Seligman et al. 2009). Entsprechend wird davon ausgegangen, dass eine gelungene Auseinandersetzung mit den Erwartungen von Patient:innen die therapeutische Beziehung stärken kann (Dew und Bickman 2005; Fende Guajardo und Anderson 2007).

Noch nicht ausreichend erforscht ist, inwiefern sich die subjektiven Konzepte zur Wirkweise von Psychotherapie von therapieunerfahrenen Menschen von denjenigen Annahmen unterscheiden, die einer professionellen Sichtweise entsprechen. Es wird davon ausgegangen, dass Psychotherapie über ein Zusammenspiel (Pfammatter und Tschacher 2012, 2016; Tschacher et al. 2014) allgemeiner Wirkfaktoren (Grawe 1995; Jørgensen 2004; Lambert und Ogles 2004) und schulenspezifischer Therapietechniken wirkt (Gumz et al. 2017; Pfammatter 2012, 2016). Insbesondere die mediierenden
Prozesse, wie kognitive Veränderungen und die therapeutische Beziehung, erscheinen bedeutsam (Baier et al. 2020). Während es also Forschung zur Frage gibt, wie Veränderungen in der Psychotherapie zustande kommen, existieren nur wenige Untersuchungen dazu, welche Annahmen Menschen ohne Psychotherapieerfahrungen diesbezüglich haben. Tzur Bitan und Abayed (2020) beschrieben auf der Basis von Mixed-methodsAnsätzen, dass sich Erwartungen an die Wirkung von Psychotherapie auf 7 Skalen erfassen lassen: Erwartungen an den Aufbau einer positiven Beziehung, inwieweit die therapeutische Beziehung verbal thematisiert wird, inwieweit unterdrückte Inhalte exploriert werden, ob die Möglichkeit besteht, sensible Inhalte offen zu teilen, ob spezifische emotionale Probleme bearbeitet werden können, ob Resilienz gefördert wird und inwieweit man Werkzeuge für die kognitive Kontrolle erhält. Während Therapeut:innen i.Allg. stärker an die Wirksamkeit der therapeutischen Beziehung und deren verbaler Thematisierung glaubten, schienen Personen ohne Therapieerfahrung und Patient:innen zuversichtlicher, dass konkrete Hinweise und Werkzeuge in der Therapie wirken (Tzur Bitan und Abayed 2020). Die Autor:innen wiesen auf kulturelle Aspekte der Fragestellung hin: Die Erwartungen der Therapeut:innen sind im Kontext einer psychodynamisch-geprägten Therapietradition Israels zu betrachten. Entsprechende Untersuchungen in anderen Regionen stehen aus (Tzur Bitan und Abayed 2020). Ein Hinweis darauf, dass die angenommene Wirkung von Psychotherapie unter deutschen Therapeut:innen sich an ähnlichen Dimensionen orientiert, findet sich in der Studie von Pfammatter und Tschacher (2016). Eine offene Erhebung zur Sichtweise von Personen ohne Therapieerfahrung liegt bisher jedoch nicht vor.

Dem sprachlichen Austausch kommt in allen Therapieschulen und der populären Darstellung von Psychotherapie eine wichtige Rolle zu (Gumz et al. 2015; von Sydow 2007; Orchowski et al. 2006). Dennoch existiert das Vorurteil, in der Psychotherapie werde viel geredet, aber es geschehe eigentlich nichts (LöfflerStastka et al. 2010). Um sprachliche Me- diatoren der Veränderung und sprachliche Wirkfaktoren zu erfassen, wurden 23 Therapeut:innen (Verhaltenstherapie [VT], tiefenpsychologisch-fundierte Psychotherapie [TP], analytische Psychotherapie [AP]) und 16 Patient:innen (VT, TP, AP) dazu interviewt, welche kurativen Aspekte des Miteinanderredens sie in der Therapie erlebten (Marx et al. 2021; Wulf et al. 2021). Die Therapeut:innen und Patient:innen beschrieben ein breites Spektrum: Beispielsweise äußerten sie, dass durch das Miteinanderreden das Erleben differenziert und strukturiert wird, emotionale Unterstützung und Resonanz erlebt werden sowie Handlungsfähigkeit erzeugt wird (Marx et al. 2021; Wulf et al. 2021). Unbekannt ist jedoch, welche Erwartungen Personen ohne Therapieerfahrung konkret an das Miteinanderreden in der Psychotherapie haben, und wie sie sich therapeutische Veränderungen erklären.

\section{Ziel der vorliegenden Studie}

Ziel der Studie ist es, ein besseres Verständnis für gegenwärtige Annahmen zu therapeutischen Prozessen zu erhalten und so weitere Ansatzpunkte für Kommunikationskampagnen, die Entstigmatisierung von Psychotherapie und die therapeutische Auseinandersetzung mit Erwartungen zu erarbeiten.

Die Forschungsfrage lautet: Welche Aspekte prägen das Bild, dass Menschen ohne Therapieerfahrung von der Wirkung des Miteinanderredens in der Therapie haben?

\section{Methoden}

\section{Studiendesign und Datenerhebung}

Es handelt sich um eine querschnittliche Mixed-methods-Studie in Form einer qualitativen Kategorienbildung mit anschließender quantitativer Häufigkeitsanalyse. Die Daten wurden im Sommer 2021 anonym mithilfe der Online-Plattform SoSci Survey (Leiner 2021) erhoben. Die Studieninfos wurden auf der ersten Seite der Untersuchung digital zur Verfügung gestellt, anschließend wurde die Zustimmung zur Studienteilnahme digital eingeholt. Eine Aufwandsentschädigung gab es nicht. 
Tab. 1 Soziodemografische Daten der Teilnehmer:innen

\begin{tabular}{|l|l|l|}
\hline \multirow{2}{*}{ Soziodemografisches Merkmal } & \multicolumn{2}{l|}{ Teilnehmer:innen } \\
\cline { 2 - 3 } \multicolumn{2}{|l|}{ Anzahl $(n)$} & Anteil (\%) \\
\hline Geschlecht & 18 & 8 \\
\hline Männlich & 207 & 92 \\
\hline Weiblich & \multicolumn{2}{l}{} \\
\hline Beziehungsstatus & 155 & 68,9 \\
\hline Ja & 67 & 29,8 \\
\hline Nein & 3 & 1,3 \\
\hline Ich bin mir nicht sicher & \multicolumn{2}{|l}{} \\
\hline Kinder & 17 & 7,6 \\
\hline Ja & 208 & 92,4 \\
\hline Nein & \multicolumn{2}{|l}{} \\
\hline Staatsangehörigkeit & 215 & 95,6 \\
\hline Deutsch, Österreich, Schweiz & 10 & 4,4 \\
\hline Eine andere oder noch eine weitere & \multicolumn{2}{|l}{} \\
\hline Höchster Bildungsabschluss & 1 & 0,4 \\
\hline Ohne Abschluss & 8 & 3,6 \\
\hline Hauptschule/Volksschule/Realschule & 75 & 33,3 \\
\hline Abitur/Matura & 37 & 16,4 \\
\hline Berufsschule/Meisterschule & 103 & 45,7 \\
\hline Studium & 1 & 0,4 \\
\hline Sonstige & \multicolumn{2}{|l}{} \\
\hline
\end{tabular}

Rekrutiert wurden die Teilnehmer:innen online über soziale Medien (Facebookgruppen, Instagram) sowie über private Netzwerke. In den sozialen Netzwerken war die Information nur für Personen sichtbar, die bereits Mitglied in den Facebookgruppen waren, mit Personen befreundet waren, die die Infos teilten oder ihnen folgten. Der Teilnahmeaufruf bestand aus zwei kurzen Sätzen zur Studie, dem Link zur Teilnahme und der Bitte, die Untersuchung auch an Familie, Freunde und Bekannte weiterzuleiten. In den forschungsaffinen Facebookgruppen wurde an dieser Stelle bereits explizit betont, dass Psychologiestudierende nicht an der Studie teilnehmen sollten.

Eingeschlossen wurden Personen, die mindestens 18 Jahre alt waren. Ausgeschlossen wurden Personen, die eigene Psychotherapieerfahrung, Erfahrung in psychotherapeutischer Beratung (Paarund Familientherapie, psychologische Beratungsstellen) hatten und/oder beruflich einer psychologischen oder psychotherapeutischen Tätigkeit oder einem Psychologiestudium nachgingen.

\section{Stichprobe}

Der Einladung zur Online-Erhebung folgten 470 Personen. Zu Beginn der Online-Erhebung wurden die Ein-und Ausschlusskriterien abgefragt. Es wurden 245 Personen aufgrund ihrer therapeutischen Vorerfahrung, ihrer psychologischen beruflichen Tätigkeit oder ihres Psychologiestudiums ausgeschlossen. In die Studie eingeschlossen wurden 225 Personen (• Tab. 1); die Daten dieser Personen gingen in die vorliegende Auswertung ein. Der Altersrange betrug 19 bis 91 Jahre. Das durchschnittliche Alter der Teilnehmer:innen betrug 27,53 Jahre (SD $\pm 9,93$ Jahre).

\section{Instrumente und offene Fragestellung}

Die Erhebung beinhaltete einen Fragebogen zu soziodemografischen Daten sowie eine Frage zur Vorerfahrung mit Psychotherapie und Beratung. Es wurde zudem die offene Frage: "Wie und auf welche Art und Weise hilft das Miteinanderreden in der Psychotherapie?" gestellt, die schriftlich beantwortet werden sollte. Die Formulierung der offenen Frage ist angelehnt an ein von der Heigl-Stiftung ge- fördertes Forschungsprojekt, in dem die kurativen Funktionen des Miteinanderredens in der Psychotherapie "bottom-up" untersucht wurden. Hier wurden $16 \mathrm{~Pa}$ tient:innen (Wulf et al. 2021) und 23 Therapeut:innen (Marx et al. 2021) live dazu interviewt, welche kurativen Aspekte des Miteinanderredens sie in der Therapie erlebten. Mit der Frage sollten in der vorliegenden Studie gezielt grundlegende Annahmen über Psychotherapie erfasst werden, und nicht etwa, welche Faktoren eine Person für sich als wirksam annimmt. Die Hypothese war, dass die grundlegenden Annahmen den Diskurs stärker beeinflussen, also sich zum Beispiel darauf auswirken, ob jemand einer nahestehenden Person eine Psychotherapie empfehlen würde. Diese Unterscheidung entspricht etwa der Trennung in selbstbezogene Stigmatisierung und wahrgenommene Stigmatisierung von Psychotherapie/psychischer Gesundheit, die ebenfalls als zwei Facetten getrennt voneinander betrachtet werden (Jennings et al. 2015; Vogel et al. 2007).

\section{Datenanalyse}

Die Daten wurden mithilfe der qualitativen Inhaltsanalyse ausgewertet (Mayring 2015). Für die Analysen wurde das Programm MAXQDA Analytics Pro 2020 (Fa. VERBI GmbH, Berlin) verwendet. Die Darstellung orientiert sich an den "Standards for reporting qualitative research" ${ }^{\prime \prime} \mathrm{O}^{\prime}$ Brien et al. 2014): Im ersten Schritt wurden die Codes/Kategorien von einem Primärteam (2 Studierende im Master-Studiengang Psychologie: B.J., A.N.) induktiv gebildet und zu Ober- und Subkategorien zusammengefasst. Dabei wurde berücksichtigt, dass inhaltlich unterscheidbare Teilaspekte der Antwort einer Person mit verschiedenen Codes versehen werden konnten (Mehrfachcodierung). Im zweiten Schritt wurden die Zuordnungen der Aussagen zu den Kategorien sowie die Benennung der Kategorien vom Sekundärteam (F.K., S.W.: Doktoranden und Psychotherapeut:innen in Ausbildung) überprüft und leicht überarbeitet. Im dritten Schritt führte A.G. (Professorin für Psychosomatik und Psychotherapie) ein weiteres Auditing durch. Diskrepanzen wurden markiert, hieran anschließend fand eine Konsensfindung im Gesamtteam statt. Im letzten Schritt 
wurden Häufigkeitsanalysen durchgeführt. Mögliche Unterschiede hinsichtlich der inhaltlichen Qualität in Abhängigkeit vom Geschlecht und Bildungshintergrund wurden ebenfalls mithilfe von MAXQDA Analytics Pro 2020 (Fa. VERBI GmbH, Berlin; VERBI 2020) geprüft. Dazu wurden Mixed-methods- und "Code-relations"Funktionen in der Software verwendet.

Die Zuverlässigkeit und intersubjektive Nachvollziehbarkeit wurden zudem durch die Diskussion der Studienergebnisse in der Forschungsgruppe geprüft. Vorannahmen und Erwartungen der Projektmitarbeiter waren: B.J. erwartete, dass aus der Perspektive der Personen ohne Psychotherapieerfahrung das Miteinanderreden zu einer erheblichen emotionalen Entlastung der Patient:innen beiträgt. A.N. und A.G. gingen davon aus, dass die Teilnehmer:innen v.a. als relevant erachten, in der Therapie ohne Bewertung alles erzählen zu können, sich öffnen zu dürfen und dort professionelle Tipps und Ratschläge zu bekommen, die innen weiterhelfen. F.K. erwartete, dass die Befragten sich vom therapeutischen Miteinanderreden v.a. neue Sichtweisen erhofften, und eine stärkere Klarheit darüber, welcher Umgang mit empfundenen Leid richtig sei. S.W. erwartete, dass Personen ohne Therapieerfahrung sich durch das Miteinanderreden emotionale und handlungsbezogene Unterstützung erhoffen und sie ihr eigenes Erleben klarer verstehen.

\section{Ergebnisse}

Die Textlänge betrug je Antwort in Wörtern $M=14,57(S D \pm 11,79)$ und in Zeichen, einschließlich Leerzeichen, $M=97,44$ (SD $\pm 77,66$ ) bei einem Range von einem Wort (3 Zeichen, einschließlich Leerzeichen) bis 61 Wörtern (430 Zeichen, einschließlich Leerzeichen).

Die inhaltliche Qualität der Antworten wurde vom Primärteam in MAXQDA codiert. Eine hohe Qualität der Antworten wurde codiert, wenn tiefgehend und differenziert auf die gestellte Frage geantwortet wurde. Von einer niedrigen Qualität wurde ausgegangen, wenn oberflächlich bzw. pauschalisierend geantwortet wurde. Insgesamt zeigte sich, dass Antworten mit niedriger inhaltlicher Qualität kurz waren. Der umgekehrte Schluss konnte jedoch nicht gezogen werden, da auch viele kurze Antworten der Teilnehmenden eine hohe inhaltliche Qualität aufwiesen.

Um das Antwortverhalten näher zu charakterisieren, wurde zudem die grafische Darstellung der Code relations (Kodierungszusammenhänge) mit MAXQDA vom Forschungsteam überprüft. Hier gab es wenige Überschneidungen der inhaltlichen Qualität (Codierung der inhaltlichen Differenziertheit) der Antworten mit dem Bildungsstatus. Bezüglich des Geschlechts zeigte sich in den visuellen Code-relationsAnalysen, dass die Antworten der männlichen Personen häufig kurz ausfielen und die inhaltliche Qualität der Antworten häufig als gering codiert wurde. Hier ist jedoch die unterdurchschnittliche Repräsentation der 18 männlichen Teilnehmer im Vergleich zu weiblichen Teilnehmerinnen $(n=207)$ zu berücksichtigen.

Aus der induktiven Analyse der offenen Frage "Wie und auf welche Art und Weise hilft das Miteinanderreden in der Psychotherapie?" heraus wurden innerhalb des Codesystems 4 Oberkategorien entwickelt, die jeweils verschiedene Subkategorien einschließen (•Tab. 2).

Die Teilnehmer:innen beschrieben, dass sie das Erleben der therapeutischen Beziehung als wirksam erwarteten (129 Codierungen in der gesamten Stichprobe; entspricht der zweitgrößten Anzahl der Nennungen aller Oberkategorien), etwa dass die Etablierung eines geschützten Raums und das Vertrauen dabei eine große Rolle spielen (26 Codierungen). Ebenso häufig wurde erwartet, Verständnis und Anerkennung im Gespräch zu erfahren (26 Codierungen) und, dass durch die persönliche verbale Interaktion Alleinsein und Isolation gelindert werden (26 Codierungen). Dass der/die Behandler:in eine neutrale Person sei und der objektive Blick helfe, wurde mehrfach angenommen (16 Codierungen). In einem ähnlichen Kontext standen die Annahmen, dass Behandler:innen weder verurteilen noch bewerten (13 Codierungen) und Patient:innen durch das Miteinanderreden Aufmerksamkeit und Interesse erfahren (9 Codierungen). Zudem ließen sich erlebte Unterstützung (8 Codierungen) und das Erleben gemeinsamer Arbeit in der Therapie (5 Codierungen) als formulierte Erwartungen ausmachen.
In der zweiten Oberkategorie wurde thematisiert, wie das Miteinanderreden aus Sicht von Lai:innen Erleichterung verschafft (45 Codierungen; entspricht der dritthäufigsten Oberkategorie). Dies wurde inhaltlich oft so konzeptualisiert, dass erwartet wurde, Ballast loszuwerden und in sich hineingefressene Dinge/Probleme von der Seele reden können.

Die dritte Oberkategorie beinhaltete Subkategorien, die den Erkenntnisgewinn beleuchten, den das Miteinanderreden erbringen kann. Es war die Oberkategorie mit den meisten Subkategorien und Codierungen (131). Vor allem der erwartete Perspektivwechsel spielte eine große Rolle (29 Codierungen). Auch wurde das Aussprechen von Gedanken, Gefühlen und Erfahrungen als besonders hilfreich empfunden, um Klarheit zu gewinnen und die Dinge auf den Tisch zu bringen (26 Codierungen). In eine ähnliche Richtung wies die Subkategorie Aufarbeitung/Verarbeitung (19 Codierungen), wobei hier mehrfach die Auseinandersetzung mit (verdrängten) Erfahrungen (in der Kindheit) genannt wurde. Sich beim Miteinanderreden den Dingen und sich selbst bewusst zu werden, wurde erwartet (1 Codierungen 7), ebenso wie (Selbst-)Reflexion (14 Codierungen) und die Erforschung von Ursachen (12 Codierungen).

Wie aus Sicht von Personen ohne Therapieerfahrung der sprachliche Austausch von Patient:in und Therapeut:in Veränderungen ermöglichen kann, wurde in der vierten Oberkategorie zusammengefasst. Dies war die Kategorie mit den wenigsten Codierungen (28). Zum einen ging es darum, Lösungsansätze zu erarbeiten bzw. Probleme konkret zu lösen (17 Codierungen). Zum anderen wurde vermutet, dass im Dialog aufgezeigt wird, wie man mit Problemen bzw. seiner Erkrankung umgehen kann (11 Codierungen).

\section{Diskussion}

\section{Interpretation der Ergebnisse}

Ziel der vorliegenden qualitativen Untersuchung war es, ein besseres Verständnis über die subjektiven Erwartungen zu den kurativen Funktionen des Miteinanderredens aus Sicht von Personen 
Tab. 2 Kategoriensystem zu den Erwartungen der Studienteilnehmer:innen $(n=225)$ an das Miteinanderreden in der Psychotherapie mit Häufigkeit der Nennung (absolut und in Prozent der Teilnehmenden) sowie illustrativen Aussagen

\begin{tabular}{|c|c|c|c|c|}
\hline \multicolumn{2}{|c|}{ Kategorie } & $\begin{array}{l}\text { Anzahl }(n) \text { der } \\
\text { Codierungen }\end{array}$ & $\begin{array}{l}\text { Anteil (\%) der } \\
\text { Teilnehmer:innen }\end{array}$ & \multirow[t]{2}{*}{ Illustrative Aussagen von Teilnehmer:innen } \\
\hline 1 & Therapeutische Beziehung er & ben $(n=129 \mathrm{Coc}$ & rungen) & \\
\hline 1.1 & Geschützter Raum, Vertrauen & 26 & 11,6 & $\begin{array}{l}\text { Sobald man dem Gegenüber vertraut, öffnet man sich mehr und } \\
\text { kann sich mehr "fallen“ lassen. Ich glaube, dass allein das bereits } \\
\text { hilft }\end{array}$ \\
\hline 1.2 & $\begin{array}{l}\text { Verständnis und Anerken- } \\
\text { nung }\end{array}$ & 26 & 11,6 & $\begin{array}{l}\text { Das Reden ist sehr bedeutend, da so gegenseitiges Verständnis } \\
\text { entstehen kann }\end{array}$ \\
\hline 1.3 & $\begin{array}{l}\text { Neutrales/objektives Gegen- } \\
\text { über }\end{array}$ & 16 & 7,1 & $\begin{array}{l}\text { Zuhören, Fachlichkeit, keine emotionale Reaktion der Thera- } \\
\text { peutin auf Beziehungsebene, was bei Familie und Freundschaft } \\
\text { automatisch der Fall ist }\end{array}$ \\
\hline 1.4 & $\begin{array}{l}\text { Nicht verurteilt/bewertet } \\
\text { werden }\end{array}$ & 13 & 5,8 & $\begin{array}{l}\text { Man kann seine Sorgen einer unabhängigen Person erzählen, die } \\
\text { einen nicht verurteilt }\end{array}$ \\
\hline 1.5 & $\begin{array}{l}\text { Aufmerksamkeit und Interes- } \\
\text { se }\end{array}$ & 9 & 4,0 & $\begin{array}{l}\text { Ich denke, Reden ist mit der wichtigste Punkt. Es drückt die An- } \\
\text { teilnahme und das Interesse an dem Menschen aus }\end{array}$ \\
\hline 1.6 & Alleinsein/Isolation lindern & 26 & 11,6 & $\begin{array}{l}\text { Ich denke, Reden und dass einem jemand zu hört, Verständnis } \\
\text { aufbringt, ist sehr wichtig, um sich nicht allein gelassen zu fühlen }\end{array}$ \\
\hline 1.7 & Unterstützung & 8 & 3,6 & $\begin{array}{l}\text { Man hat eine Person, an die man sich wenden kann: wie ein Fels } \\
\text { in der Brandung }\end{array}$ \\
\hline 1.8 & Gemeinsam arbeiten & 5 & 2,2 & Gemeinsames Erarbeiten der Probleme und Hilfestellung \\
\hline 2 & \multicolumn{4}{|c|}{ Erleichterung verschaffen ( $n=45$ Codierungen) } \\
\hline 2.1 & $\begin{array}{l}\text { Ballast loswerden, von der } \\
\text { Seele reden }\end{array}$ & 45 & 20,0 & $\begin{array}{l}\text { Oft tut es den Patienten schon gut, sich Sorgen und Gedanken } \\
\text { von der Seele zu reden und nicht alles in sich hineinzufressen }\end{array}$ \\
\hline 3 & \multicolumn{4}{|c|}{ Erkenntnis gewinnen ( $n=131$ Codierungen) } \\
\hline 3.1 & $\begin{array}{l}\text { Professioneller Rat und Wis- } \\
\text { sen }\end{array}$ & 9 & 4,0 & $\begin{array}{l}\text { Jemand ist da, der einem Schritt für Schritt einen Weg oder Lö- } \\
\text { sungsansatz zeigt }\end{array}$ \\
\hline 3.2 & Perspektivwechsel & 29 & 12,9 & $\begin{array}{l}\text { Andere Sichtweisen können einem helfen, auch für sich selbst die } \\
\text { Problematik anders zu sehen und an sie ranzugehen }\end{array}$ \\
\hline 3.3 & Aussprechen schafft Klarheit & 26 & 11,6 & $\begin{array}{l}\text { Oft bemerkt man erst beim Aussprechen von Dingen, dass es } \\
\text { einem ggf. nicht gut geht, bzw. was einen stört }\end{array}$ \\
\hline 3.4 & Aufarbeitung/Verarbeitung & 19 & 8,4 & Sehr viel! Aufarbeiten der Erlebnisse ist sehr wichtig. Verarbeitung \\
\hline 3.5 & Bewusst werden, erkennen & 17 & 7,6 & $\begin{array}{l}\text { Ich glaube, dadurch werden einem seine Gefühle und Gedanken } \\
\text { erst richtig bewusst }\end{array}$ \\
\hline 3.6 & Reflexion & 14 & 6,2 & Man fängt an, sich selbst zu reflektieren \\
\hline 3.7 & Ursachenforschung & 12 & 5,3 & $\begin{array}{l}\text { Es hilft dabei, den Ursachen des Problems auf die Spur zu kom- } \\
\text { men }\end{array}$ \\
\hline 3.8 & $\begin{array}{l}\text { Mit den Dingen/Problemen } \\
\text { konfrontiert werden }\end{array}$ & 9 & 4,0 & $\begin{array}{l}\text { Man wird als Patient gezwungen, über Dinge zu sprechen, die } \\
\text { man sonst verdrängt }\end{array}$ \\
\hline 3.9 & Die Situation akzeptieren & 5 & 2,2 & Sehr, da die Betroffenen ihr Problem anerkennen \\
\hline 4 & \multicolumn{4}{|c|}{ Veränderung ermöglichen ( $n=28$ Codierungen) } \\
\hline 4.1 & $\begin{array}{l}\text { Lösungsansätze erarbeiten, } \\
\text { Probleme lösen }\end{array}$ & 17 & 7,6 & $\begin{array}{l}\text { Der Psychotherapeut kann neue Handlungswege/Optionen etc. } \\
\text { aufzeigen, die Betroffenen helfen können }\end{array}$ \\
\hline 4.2 & $\begin{array}{l}\text { Mit Problemen/Erkrankung } \\
\text { umgehen lernen }\end{array}$ & 11 & 4,9 & Neue Wege finden, mit Problemen umzugehen \\
\hline
\end{tabular}

ohne Psychotherapieerfahrung zu erhalten. Die inhaltsanalytische Auswertung zeigt, dass die Teilnehmer:innen vielfältige und differenzierte Perspektiven darauf haben, wie das Miteinanderreden im psychotherapeutischen Kontext wirken kann. Die Teilnehmer:innen erwarteten die Wirksamkeit des Miteinanderredens in der Psychotherapie in Bezug auf die vier Hauptkategorien 1) Therapeutische Beziehung erleben, 2) Erleichterung verschaffen, 3) Erkenntnis gewinnen sowie 4) Veränderungen ermöglichen. Von diesen Hauptkategorien wurden Aspekte des Erkenntnisgewinns am häufigsten benannt. Hier werden folgende Erwartungen in Bezug auf das Miteinanderreden besonders oft erwähnt: Einen Perspek- tivwechsel erhalten und Klarheit schaffen. Am zweithäufigsten fanden Aspekte der Hauptkategorie Therapeutische Beziehung erleben Erwähnung. Vertrauen sowie Verständnis und Anerkennung durch den/die Therapeut:in werden als besonders bedeutend erachtet. Am dritthäufigsten wurde die Hauptkategorie $E r$ leichterung verschaffen beschrieben. Hier 
sprachen die Befragten davon, Ballast loszuwerden und in sich hineingefressene Dinge und Probleme von der Seele reden zu können. Von allen Subkategorien der gesamten Untersuchung wurde diese mit Abstand am häufigsten genannt. Sie wurde sogar öfter erwähnt als Facetten der Hauptkategorie Veränderungsmöglichkeiten.

Insgesamt scheinen Personen ohne Psychotherapieerfahrung sehr differenzierte Erwartungen an das Miteinanderreden in der Psychotherapie zu haben. Psychotherapie scheint in den Augen der Befragten nicht wirkungsloses Gerede zu sein, sondern durch das therapeutische Gespräch wird aus Sicht der Befragten eine Vielzahl allgemeiner Wirkfaktoren umgesetzt bzw. werden mediierende Veränderungsprozesse initiiert. Viele in der Forschungsliteratur erwähnte allgemeine Wirkfaktoren der Psychotherapie und Mediatoren der Veränderung sind in der vorliegenden Untersuchung auch in den Konzepten der Personen ohne Psychotherapieerfahrung vorhanden: Von den allgemeinen Wirkfaktoren fanden sich beispielsweise die therapeutische Beziehung (Pfammatter und Tschacher 2016; Omer und London 1989; Grencavage und Norcross 1990), Einsicht (Tschacher et al. 2014), Handlungsfaktoren (Lambert und Ogles 2004), Perspektivenübernahme im Sinne von Mentalisierungsfähigkeit (Pfammater und Tschacher 2016) und Problembewältigung (Grawe 1995). Anders als in gängigen Systematiken der allgemeinen Wirkfaktoren (z.B. Grawe 1995) werden in der vorliegenden Untersuchung sowie in den in vorangegangenen Untersuchungen zu den Wirkfaktoren des Miteinanderredens aus Patienten- bzw. Therapeutenperspektive (Marx et al. 2021; Wulf et al. 2021) motivationale Klärung und Intentionsänderung nicht erwähnt. Dies könnte darauf hinweisen, dass diese kurative Funktion nicht direkt mit dem Miteinanderreden assoziiert wird.

Insbesondere die Hauptkategorien Erkenntnisgewinn und Erleben in der therapeutischen Beziehung erscheinen differenziert und bedeutsam. Dies steht im Einklang mit einer Studie mit britischen Jugendlichen, die vor einer Psychoanalyse besonders hohe Erwartungen an die affektive Beziehung zur Therapeutin/zum
Therapeuten, an das Zuhören, das Angenommenwerden sowie an das ausführliche Gespräch und die Möglichkeit des Nachdenkens äußerten (Bury et al. 2007). Auch die Personen ohne Psychotherapieerfahrung im psychoanalytisch-geprägten Israel (Tzur Bitan und Abayed 2020) hatten ähnliche Erwartungen an den Aufbau einer positiven Beziehung, die Exploration unterdrückter Inhalte und die Möglichkeit, sensible Inhalte offen zu teilen. Allerdings wurden Werkzeuge kognitiver Kontrolle und das Fördern von Resilienz anders als bei Tzur Bitan und Abayed (2020) von den Personen in der vorliegenden Untersuchung kaum thematisiert. Da sich das Studiendesign und die genaue Fragestellung (Fokus auf das Miteinanderreden/Therapie allgemein) in diesen beiden Untersuchungen jedoch unterschieden, sollte ein Vergleich dieser Befunde zurückhaltend interpretiert werden. Die erwähnten Unterschiede hängen möglicherweise weniger mit den kulturellen Besonderheiten zusammen, sondern eher damit, dass Resilienz und kognitive Kontrolle eher langfristigere Ziele auf Ebene des Makro-Outcome sind, die nicht unmittelbar mit dem Miteinanderreden in Zusammenhang gebracht werden.

Die subjektiven Konzepte über die kurativen Funktionen des Miteinanderredens in der vorliegenden Studie waren denen derTherapeut:innen in der Studie von Marx et al. (2021) und denen der Patient:innen (Wulf et al. 2021) sehr ähnlich. Dies deckt sich mit den Ergebnissen von Tzur Bitan und Abayed (2020), die berichteten, dass sich die subjektiven Konzepte über die Wirkung von Psychotherapie zwischen Patient:innen und Personen ohne Therapieerfahrung statistisch nicht signifikant unterscheiden. Interessant ist, dass Erwartungen an das Miteinanderreden in der Psychotherapie spezifisch zu sein scheinen. So erwähnten jeweils mindestens $40 \%$ der Befragten keine Beziehungsaspekte oder keine Aspekte des Erkenntnisgewinns. Dies könnte ein Hinweis auf die Bedeutsamkeit der individuellen Erwartungen an die Psychotherapie sein. Anknüpfend wäre sowohl theoretisch als auch für die praktische therapeutische Arbeit interessant, was von wem unter welchen Bedingungen erwartet wird, und ob sich die Erwartungen an verschiedene Therapieformen (VT, TP, AP, ST) unterscheiden. Dies böte die Möglich- keit, gezielt mit verschiedenen Erwartungen umzugehen und etwaige problematische Diskrepanzen (Seligman et al. 2009) früh zu bearbeiten.

\section{Methodische Stärken und Schwächen}

Für eine qualitative Studie ist die Stichprobengröße groß und erlaubte es uns, vielfältige Perspektiven einzubeziehen. Die Befragung war kurz gehalten und somit niedrigschwellig auch für solche Teilnehmer:innen zu beantworten, die womöglich von längeren Umfragen oder ausführlicheren Interviews abgeschreckt worden wären. Gleichzeitig waren die Antworten im Vergleich zu Interviews weniger tiefgehend, und Nachfragen waren für Teilnehmende nicht möglich.

Voraussichtlich beeinflusst durch die Rekrutierungsstrategie, die versuchte, Freiwillige vorrangig über Social-MediaKanäle zu erreichen, ist die Stichprobe keinesfalls repräsentativ. Die hier herausgearbeiteten, erwarteten Wirkfaktoren stammen vorrangig von jungen deutschen Frauen, die mehrheitlich gut ausgebildet und (noch) kinderlos sind. Ihre Antworten lassen sich somit nicht verallgemeinern. Diese Einschränkung ist typisch für vergleichbare Studien. In der im Vergleich zur vorliegenden Untersuchung ähnlich aufgebauten Studie von Tzur Bitan und Lazar (2019) waren etwa $71 \%$ der Teilnehmenden weiblich und mit einem mittleren Alter von 25,56 vergleichsweise jung. Auch für die - im Hinblick auf die Fallzahlen vergleichbare Studie von Tzur Bitan und Abayed (2020) gelten Einschränkungen im Hinblick auf die Generalisierbarkeit: So waren dort unter den Therapieunerfahrenen ebenfalls $90 \%$ der Teilnehmenden weiblich, das mittlere Alter betrug 22,5 Jahre, und der Bildungsgrad war hoch. Angesichts der oft problematischen Rolle von Maskulinitätsnormen (Chatmon 2020) auf die Wahrnehmung und Stigmatisierung des Themas sollte weitere Forschung stärker auf die Repräsentanz von Männern achten.

Auch können weitere Verzerrungseffekte nicht ausgeschlossen werden, da etwa Personen mit geringerer sprachlicher Kompetenz möglicherweise weniger für die Umfrage akquiriert werden konnten. 


\section{Ausblick}

Insgesamt leistet die Erhebung den ersten Schritt in Richtung eines besseren Verständnisses der Erwartungen an Psychotherapie und insbesondere an das Miteinanderreden. Da v.a. jungen Frauen rekrutiert wurden, stehen weitere Untersuchungen in repräsentativeren Stichproben aus. Angesichts unterschiedlicher sozialer Kontexte und der zu erwartenden unterschiedlich geführten Diskurse um Gesundheit und Psyche erscheint eine zielgruppenspezifische Auseinandersetzung für ein Verständnis gesellschaftlicher Konstruktionen von Psychotherapie hilfreich. Auch die Frage, ob sich Annahmen über die Entstehung psychischer Störungen darauf auswirken, welche Erwartungen an Psychotherapie Personen ohne Therapieerfahrung entwickeln, scheint beispielsweise im Hinblick auf die Formulierung von Anti-Stigma-Kampagnen und Psychoedukation ein möglicher nächster Schritt. Interessant erscheint zudem mitzuerfassen, woher die Personen ihr Wissen über Psychotherapie haben, und ob sie selbst glauben, dass innen das Miteinanderreden in der Psychotherapie helfen könnte, bzw. ob sie selbst psychotherapeutische Hilfe in Anspruch nehmen würden. Für die zukünftige Untersuchung subjektiver Konzepte von Personen ohne Therapieerfahrungen könnte zudem bereichernd sein, welche Erwartungen dazu beitragen, unterschiedliche Formen von Psychotherapie als hilfreiche Option anzusehen oder selbst wahrzunehmen, und ob sich die Erwartungen an die Funktionen des Miteinanderredens bei verschiedenen Therapieschulen unterscheiden.

Perspektivisch sollten die Erwartungen an das Miteinanderreden in der Psychotherapie zudem mit den erlebten kurativen Funktionen des Miteinanderredens aus Sicht erfahrener Patient:innen verglichen werden. Dies erscheint insbesondere im Hinblick auf die optimale Aufklärung über die Wirkweise von Psychotherapie vielversprechend.

Ferner können künftig Erwartungsunterschiede zwischen Personen mit und ohne Therapieerfahrung sowie Psychotherapeut:innen untersucht werden. Hier erscheint plausibel, dass etwa der Faktor "Veränderung ermöglichen" durch entsprechendes Fachwissen bei Therapeut:innen stärker ausdifferenziert beschrieben wird und sich auch bezüglich der Erwartungen Schulenunterschiede finden, vergleichbar zu den retrospektiv von Marx et al. (2021) berichteten Ergebnissen. Auch die zukünftigen Erwartungen von Personen mit Therapieerfahrung scheinen untersuchenswert zu sein, wobei mutmaßlich von vergangenen Erlebnissen auf zukünftige Erwartungen geschlossen wird und womöglich aus Sicht der Autor:innen die Rolle von kritischen Momenten interessiert (Gumz 2012).

\section{Fazit für die Praxis}

- Personen ohne Psychotherapieerfahrung haben differenzierte und individuell spezifische Annahmen darüber, wie das Miteinanderreden in der Psychotherapie heilen kann.

- Ein Austausch darüber, mit welchen Erwartungen an das therapeutische Miteinanderreden Patient:innen in eine Psychotherapie kommen, könnte zu Therapiebeginn hilfreich sein.

- Die von den Teilnehmenden meistgenannte Subkategorie ist, dass man sich in der Psychotherapie den Ballast von der Seele redet. Erwartungen an das Miteinanderreden in der Psychotherapie betrafen in der vorliegenden Stichprobe darüber hinaus häufiger den Erkenntnisgewinn und das Erleben in der therapeutischen Beziehung, weniger das Ermöglichen von Veränderungen.

\section{Korrespondenzadresse}

\section{S. Wulf}

Fachbereich Psychosomatik und Psychotherapie, Psychologische Hochschule Berlin (PHB)

Am Köllnischen Park 2, 10179 Berlin,

Deutschland

s.wulf@tp.phb.de

Danksagung. Wir bedanken uns bei allen Personen die an der Erhebung teilgenommen haben. Unser herzlicher Dank gilt auch allen Personen, die uns bei unserem Forschungsprojekt unterstützt haben, wie Anne Neuvians, Lisa Döhl, Charlotte Kaupa, Ina Peglow, Marcus Wahl, Elena Floto, Alina Göritz, Fabian Weber, Saskia Pytlik, Nicole Rosenberg, Nicole Wipplinger sowie Prof. Dr. Bianca Ksienzyk-Kreuziger.

Förderung. Die Studie zu den Wirkfaktoren des Miteinanderredens wurde gefördert durch Drittmittel der Heigl-Stiftung (30.11.2017; Projektleitung: Antje Gumz). Die Stiftung war nicht in die Studienkonzep- tion, die Datenerhebung, die Datenanalyse oder die Ergebnisinterpretation involviert.

Funding. Open Access funding enabled and organized by Projekt DEAL.

\section{Einhaltung ethischer Richtlinien}

Interessenkonflikt. S. Wulf, F. Klapprott, B. Janning und A. Gumz geben an, dass kein Interessenkonflikt besteht.

Alle Studienteilnehmer:innen erteilten vor Studieneinschluss eine informierte schriftliche Einwilligung zur Teilnahme. Für die Studie liegt ein positives Ethikvotum der Ethikkommission der Psychologischen Hochschule Berlin vor (AZ: EK2021/17)

Open Access. Dieser Artikel wird unter der Creative Commons Namensnennung 4.0 International Lizenz veröffentlicht, welche die Nutzung, Vervielfältigung, Bearbeitung, Verbreitung und Wiedergabe in jeglichem Medium und Format erlaubt, sofern Sie den/die ursprünglichen Autor(en) und die Quelle ordnungsgemäß nennen, einen Link zur Creative Commons Lizenz beifügen und angeben, ob Änderungen vorgenommen wurden.

Die in diesem Artikel enthaltenen Bilder und sonstiges Drittmaterial unterliegen ebenfalls der genannten Creative Commons Lizenz, sofern sich aus der Abbildungslegende nichts anderes ergibt. Sofern das betreffende Material nicht unter der genannten Creative Commons Lizenz steht und die betreffende Handlung nicht nach gesetzlichen Vorschriften erlaubt ist, ist für die oben aufgeführten Weiterverwendungen des Materials die Einwilligung des jeweiligen Rechteinhabers einzuholen.

Weitere Details zur Lizenz entnehmen Sie bitte der Lizenzinformation auf http://creativecommons.org/ licenses/by/4.0/deed.de.

\section{Literatur}

Avissar N (2016) Psychotherapy, society, and politics. Palgrave Macmillan, New York

Baier AL, Kline AC, Feeny NC (2020) Therapeutic alliance as a mediator of change: a systematic review and evaluation of research. Clin Psychol Rev 82:101921

Bury C, Raval H, Lyon L (2007) Young people's experiences of individual psychoanalytic psychotherapy. Psychol Psychother 80(1):79-69

Chatmon BN (2020) Males and mental health stigma. Am J Mens Health 14(4):1557988320949322

Corrigan P (2004). How stigma interferes with mental health care. Am Psychol 59(7):614-625

Dew SE, Bickman L (2005) Client expectancies about therapy. Ment Health Serv Res 7(1):21-33

FendeGuajardo JM, Anderson T (2007) An investigation of psychoeducational interventions about therapy. Psychother Res 17(1):120-127

Grawe K (1995) Grundriss einer Allgemeinen Psychotherapie. Psychotherapeut 40(3):130-145

Grencavage LM, Norcross JC (1990) Where are the commonalities among the therapeutic common factors? Prof Psychol Res Pr 21:372-378

Gumz A (2012) Kritische Momente im Therapieprozess. Psychotherapeut 57(3):256-262. https://doi. org/10.1007/s00278-012-0899-7 
Gumz A, Treese B, Marx C, Strauss B, Wendt H (2015) Measuring verbal psychotherapeutic techniques - asystematicreview of intervention characteristics and measures. Front Psychol 6:1705

Gumz A, Neubauer K, Horstkotte JK, Geyer M, Löwe B, Murray AM, Kästner D (2017) A bottomup approach to assess verbal therapeutic techniques. Development of the Psychodynamic Interventions List (PIL). PLoSONE 12(8):e182949

Jennings KS, Cheung JH, Britt TW, Goguen KN, Jeffirs SM, Peasley AL, Lee AC (2015) How are perceived stigma, self-stigma, and self-reliance related to treatment-seeking? A three-path model. Psychiatr Rehabil J38(2):109-116

Jørgensen CR (2004) Active ingredients in individual psychotherapy. Searching for common factors. Psychoanal Psychol 21:516-540

Kushner MG, Sher KJ (1991) The relation of treatment fearfulness and psychological service utilization an overview. Prof Psychol Res Pr 22(3):196-203

Lambert MJ, Ogles BM (2004) The efficacy and effectiveness of psychotherapy. In: Lambert MJ (Hrsg) Bergin and Garfield's handbook of psychotherapy and behavior change, 5. Aufl. Wiley, New York, S 139-193

Leiner DJ (2021) Sosci survey (version 2.6.00-i) [computer software]. https://www.soscisurvey. de.Zugegriffen: 15. Aug. 2021

Löffler-Stastka H, Blüml V, Ponocny-Seliger $E$, Hodal M, Jandl-Jager E, Springer-Kremser M (2010) Einstellungen von Medizinstudenten zu Psychotherapie: Veränderungen nach Unterricht über Psychische Funktionen in Gesundheit und Krankheit. Psychother Psychosom Med Psychol 60(03/04):118-125

Marx C, Benecke C, Gumz A (2021) How can talking cure? On language in psychotherapy. Forum Psychoanal 37(1):99-115

Mayring P (2015) Qualitative Inhaltsanalyse. Grundlagen und Techniken, 12. Aufl. Beltz, Weinheim

Michalopoulou LE, Welsh JA, Perkins DF, Ormsby L (2017) Stigma and mental health service utilization in military personnel: a review of the literature. Mil Behav Health 5(1):12-25

O'Brien BC, Harris IB, Beckman TJ, Reed DA, Cook DA (2014) Standards for reporting qualitative research. Acad Med 89(9):1245-1251

Omer H, London P (1989) Signal and noise in psychotherapy. The role and control of nonspecific factors. Brit J Psychiat 155(2):239-245. https://doi.org/10.1192/bjp.155.2.239

Orchowski LM, Spickard BA, McNamara JR (2006) Cinema and the valuing of psychotherapy: implications for clinical practice. Prof Psychol Res $\operatorname{Pr}$ 37(5):506-514

Pfammatter M, Tschacher W (2012) Wirkfaktoren der Psychotherapie - Eine Übersicht und Standortbestimmung. Z Psychiatr Psychol Psychother 60:67-76

Pfammatter M, Tschacher W (2016) Klassen allgemeiner Wirkfaktoren der Psychotherapie und ihr Zusammenhang mit Therapietechniken. Z Klin Psychol Psychother 45(1):1-13

Seligman LD, Wuyek LA, Geers AL, Hovey JD, Motley RL (2009) The effects of inaccurate expectations on experiences with psychotherapy. Cogn Ther Res 33(2):139-149

von Sydow K (2007) Das Image von Psychologen, Psychotherapeuten und Psychiatern in der Öffentlichkeit. Psychotherapeut 52(5):322-333

Tschacher W, Junghan UM, Pfammatter M (2014) Towards a taxonomy of common factors in

\section{How does talking to each other in psychotherapy work from the perspective of persons without experience in therapy? A mixed methods study}

Background and aim: Talking to one another is a key feature of psychotherapy, even from the perspective of people without experience in therapy. It can be assumed that persons without experience in psychotherapy develop ideas about how therapeutic talking to one another is helpful. These perceptions and preconceived expectations can influence the therapeutic interaction in psychotherapy. To gain a better understanding of the expectations of processes in psychotherapy, the images made by people without therapy experience on talking to each other in psychotherapy are examined.

Material and method: This is a mixed methods study (qualitative category formation followed by quantitative frequency analysis). A sample of 225 adults without therapy or counselling experience (mean age 27.53 years, SD \pm 9.93 , range 19-91 years, gender 207 female, 18 male) participated in the cross-sectional online survey. In addition to sociodemographic data the open-ended question "How and in what way does talking to each other help in psychotherapy?" was asked and evaluated using qualitative content analysis.

Results: The participants expected efficacy of talking to each other in psychotherapy in terms of four major categories: 1) experiencing therapeutic rapport, 2) providing relief, 3) gaining insight and 4) facilitating change. Each of these major categories were assigned between 1 and 8 subcategories. The subcategories of getting something off one's chest/getting rid of baggage and changing perspective were mentioned particularly frequently.

Discussion: The present results indicate that persons without psychotherapy experience have differentiated and individually varying assumptions about how talking to each other in psychotherapy can heal. Thus, an exchange about which of the respective expectations patients come to psychotherapy with, could be helpful.

\section{Keywords}

Expectations - Talking cure · Therapeutic relationship · Common factors · Principles of change

psychotherapy - results of an expert survey. Clin Psychol Psychother 21(1):82-96

Tzur Bitan D, Abayed S (2020) Process expectations: differences between therapists, patients, and lay individuals in their views of what works in psychotherapy.J Clin Psychol 76(1):20-30

TzurBitan D, Lazar A (2019) What do people thinkworks in psychotherapy: A qualitative and quantitative assessment of process expectations. ProfPsychol Res Pr 50:272-277

VERBI Software (2020) MAXQDA 2020 [computer software]. VERBI Software, Berlin (Verfügbar unter maxqda.com)

Vogel DL, Wade NG, Hackler AH (2007) Perceived public stigma and the willingness to seek counseling: the mediating roles of self-stigma and attitudes toward counseling. J Couns Psychol 54(1):40

Wulf S, Reuter L, Frey L, Nitzsche A, Strauß B, Gumz A (2021) How can talking cure? Curative functions of talking in psychotherapy from patients' perspective-a qualitative analysis. SPR, Heidelberg 